\title{
Acarofauna associada à vegetação espontânea de vinhedos
}

\author{
Mitefauna associated to spontaneous vegetation of vineyards
}

\section{Raquel Bottini de Moura ${ }^{\mathrm{I}}$ Fernanda de Oliveira de Andrade Bertolo ${ }^{\mathrm{I}}$ Ana Paula Ott ${ }^{\mathrm{II}}$}

\section{RESUMO}

Objetivou-se identificar a acarofauna associada à vegetação espontânea presente em vinhedos de Vitis vinifera (L.) var. Cabernet Sauvignon, Merlot e Sémillon e V. Iabrusca (L.) var. Isabel. Amostragens mensais foram realizadas de junho a setembro/2009 e junho a setembro/2010 em Caxias do Sul e de junho/2010 a junho/2011 em Sant'Ana do Livramento, RS, Brasil. Foram registrados 474 ácaros pertencentes a 14 morfoespécies, nove gêneros e cinco famílias (Phytoseiidae, Tarsonemidae, Tenuipalpidae, Tydeidae e Winterschmidtiidae). A maior abundância de ácaros ocorreu em Caxias do Sul, entretanto a maior riqueza de espécies foi registrada em Sant'Ana do Livramento. De acordo com os resultados deste trabalho, a acarofauna presente nos nove vinhedos amostrados foi registrada em sua maioria em Sida rhombifolia L. e caracterizou-se pela ocorrência dominante de Lorryia sp., seguida por Typhlodromalus aripo (Muma, 1967) e Steneotarsonemus sp..

Palavras-chave: Acari, controle biológico, manejo integrado, Vitis vinifera, V. labrusca.

\section{ABSTRACT}

The objective of the present study was to identify the mitefauna associated to the spontaneous vegetation of vineyards of Vitis vinifera var. Cabernet Sauvignon, Merlot and Sémillon and V. Iabrusca var. Isabel in the municipalities of Caxias do Sul and Sant'Ana do Livramento, Rio Grande do Sul, Brazil. Monthly samplings were accomplished during the period of June to September 2009 and June to September 2010 in Caxias do Sul and from June 2010 to June 2011 in Sant'Ana do Livramento. A total of 474 mites belonging to 14 morfospecies of nine genera and five families (Phytoseiidae, Tarsonemidae, Tenuipalpidae, Tydeidae $e$ Winterschmidtiidae) were identified. The highest abundance of acarids was registered in Caxias do Sul, however a highest species richness was registered in Sant'Ana do Livramento. Results shows that most of the mitefauna collected at the nine sampled vineyards were registered on Sida rhombifolia L.; dominant mite species were Lorryia sp., proceeded by Typhlodromalus aripo (Muma, 1967) and Steneotarsonemus sp..

Key words: Acari, biological control, integrated management, Vitis vinifera, V. Iabrusca.

\section{INTRODUÇÃO}

A preocupação com organismos antes considerados de importância secundária na cultura da videira, levou à busca por informações sobre a acarofauna em vinhedos (JOHANN et al. 2009, KLOCK et al. 2011). No Estado do Rio Grande do Sul, especificamente, tem-se registrado com frequência, danos ocasionados por ácaros fitófagos nessa cultura (FERLA \& BOTTON, 2008, SIQUEIRA et al., 2011).

A manipulação dos agroecossistemas pelo manejo de culturas de cobertura e da vegetação espontânea influencia a dinâmica populacional de inimigos naturais favorecendo o controle biológico de artrópodes fitófagos (ALTIERI et al. 2005, MONTEIRO et al., 2002). Plantas que compõem a vegetação espontânea nos agroecossistemas, têm papel na manutenção de inimigos naturais de ácaros fitófagos, devido ao fornecimento de locais de refúgio para reprodução e proteção e por prover alimento alternativo (pólen, néctar, micélio e outras presas), mantendo-os no sistema mesmo na ausência

'Programa de Pós-graduação em Fitotecnia, Universidade Federal do Rio Grande do Sul (UFRGS), Porto Alegre, RS, Brasil.

"ILaboratório de Acarologia Agrícola, Departamento de Fitossanidade, UFRGS, Av. Bento Gonçalves 7712, 91540-000, Porto Alegre, RS, Brasil. E-mail: ana.ott@ufrgs.br. Autor para correspondência. 
da presa preferencial (FERLA \& MORAES, 2002, DUSO et al., 2010).

A diversificação do agroecossistema vitícola relaciona-se à maior abundância e diversidade de ácaros predadores, em especial da família Phytoseiidae em relação à presença de ácaros fitófagos (BABAR et al., 2010, TIXIER et al., 1998) evidenciando a importância da manutenção desses habitats. Este trabalho teve como objetivo, identificar a acarofauna associada à vegetação espontânea de vinhedos de Vitis vinifera (L.) var. Cabernet Sauvignon, Merlot e Sémillon e Vitis labrusca (L.) var. Isabel.

\section{MATERIAL E MÉTODOS}

Um vinhedo de cada uma das variedades Cabernet Sauvignon, Isabel e Sémillon foi avaliado de junho a setembro durante os anos de 2009 e 2010 (oito amostragens) em Caxias do Sul (2908'19”'S, 5059'59”O) e três vinhedos de cada uma das variedades Cabernet Sauvignon e Merlot foram avaliados de junho/2010 a junho/2011 (13 amostragens) em Sant'Ana do Livramento

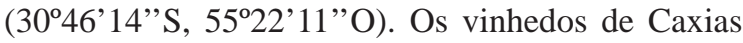
do Sul situam-se a uma altitude de $830 \mathrm{~m}$; o clima na região é classificado como $C f b$ de acordo com KÖPPEN (1948) e a temperatura média anual é de $16,5^{\circ} \mathrm{C}$. As variedades Isabel e Sémillon apresentam o sistema de condução Latada e a variedade Cabernet Sauvignon é conduzida sob o sistema Espaldeira. Em Sant'Ana do Livramento os vinhedos são conduzidos no sistema Espaldeira e encontram-se a uma altitude de 180m; o clima é classificado como Cfa (KÖPPEN, 1948) com temperatura média anual de $17,4^{\circ} \mathrm{C}$. Em todos os vinhedos foi empregado manejo fitossanitário com aplicação de acaricidas, fungicidas e herbicidas, além de roçadas periódicas.

As coletas foram realizadas mensalmente sendo sorteadas, em cada vinhedo, 10 entrelinhas e, em cada uma destas, foram coletados dois exemplares de cada uma das espécies Daucus pusillus Michx., Leucanthenum vulgare Lam. e Trifolium pratense L. em Caxias do Sul e um exemplar de cada uma das espécies Bidens pilosa L., Raphanus raphanistrum L., Sida rhombifolia L. e Solanum americanum Mill. em Sant'Ana do Livramento. As plantas foram levadas ao laboratório para a retirada dos ácaros e esses foram montados em lâminas de microscopia contendo meio de Hoyer (JEPPSON et al., 1975) e mantidos em estufa para a secagem do meio e fixação, distensão e clarificação dos espécimes. Na identificação dos espécimes foram utilizadas chaves dicotômicas (KRANTZ \& WALTER, 2009, CHANT \& MACMURTRY, 2007) e o auxílio de especialistas. Vouchers das espécies foram depositados no MCNFZB/RS e na Coleção de Referência do Laboratório de Acarologia Agrícola da UFRGS.

Na amostragem de Caxias do Sul o número de ácaros não foi relacionado às plantas da vegetação espontânea, sendo registrado apenas o número total de ácaros na vegetação espontânea por vinhedo (Tabela 1). A dominância das espécies (D) foi definida através da fórmula: $\mathrm{D} \%=(\mathrm{i} / \mathrm{t}) \cdot 100$, onde $\mathrm{i}=$ total de indivíduos de uma espécie e $\mathrm{t}=$ total de indivíduos coletados e agrupados de acordo com as categorias estabelecidas por FRIEBE (1983). Para análise da relação entre ácaros, plantas da vegetação espontânea e variedades de videira em Sant'Ana do Livramento os dados foram submetidos à análise multivariada de componentes principais com utilização do Software Past Versão 1.37 (HAMMER et al., 2001) e para análise entre diferenças numéricas foi utilizado o programa estatístico BioEstat versão 4 (AYRES et al., 2005).

\section{RESULTADOS E DISCUSSÃO}

Foram registrados 474 ácaros pertencentes a 14 espécies, nove gêneros e cinco famílias. O maior número de ácaros foi registrado em Caxias do Sul (64,5\%), sendo 70\% destes associados à variedade Sémillon (Tabela 1). JOHANN et al. (2009) registraram 920 ácaros de 43 espécies pertencentes a 11 famílias nas plantas espontâneas de vinhedos das variedades Cabernet Sauvignon e Pinot Noir em Bento Gonçalves e Candiota, RS. Nos mesmos municípios, KLOCK et al. (2011) em levantamentos na vegetação espontânea de vinhedos de Chardonnay e Merlot, registraram 602 ácaros de 18 espécies distribuídos em 13 famílias. O maior número de indivíduos, espécies e famílias registrados por esses autores em relação ao presente estudo, deve-se provavelmente ao maior número de espécies vegetais amostradas, respectivamente 63 e 59, enquanto no atual levantamento foram amostradas apenas sete espécies vegetais. Outro fator relevante é a não utilização de acaricidas e outros pesticidas durante as coletas nos estudos de Candiota e Bento Gonçalves, diferindo do manejo adotado no atual estudo o qual pode ter reduzido as populações de ácaros por serem utilizados produtos com ingredientes ativos não seletivos aos inimigos naturais. A maior presença de ácaros nas plantas presentes no vinhedo da variedade Sémillon pode ser atribuída ao manejo empregado nesse vinhedo, com menor número de aplicações 
Tabela 1 - Acarofauna presente na vegetação espontânea composta por Daucus pusillus, Leucanthenum vulgare e Trifolium pratense em vinhedos das cultivares Cabernet Sauvignon (CS), Isabel (I) e Sémillon (S) em Caxias do Sul, Rio Grande do Sul, Brasil. (n= 1440 plantas).

\begin{tabular}{|c|c|c|c|c|c|c|c|c|}
\hline Família & Espécie & CS & $\mathrm{D}$ & I & $\mathrm{D}$ & $\mathrm{S}$ & $\mathrm{D}$ & Total \\
\hline \multirow[t]{5}{*}{ Phytoseiidae } & Amblyseius herbicolus & 1 & Rar & 3 & Dom & - & & 4 \\
\hline & Amblyseius vitis & 1 & Rar & - & & - & & 1 \\
\hline & Neoseiulus tunus & 6 & Eud & 2 & Sub & 7 & Sub & 15 \\
\hline & Typhlodromalus aripo & 3 & Dom & 6 & Eud & 13 & Dom & 22 \\
\hline & Typhlodromalus sp. & - & & - & & 1 & Rar & 1 \\
\hline Tarsonemidae & Steneotarsonemus sp. & 1 & Rar & 1 & Rar & - & & 2 \\
\hline Tenuipalpidae & Brevipalpus phoenicis & 2 & Sub & 2 & Sub & - & & 4 \\
\hline Tydeidae & Lorryia sp. & 41 & Eud & 28 & Eud & 186 & Eud & 255 \\
\hline Winterschimidtiidae & Czenspinskia sp. & - & & - & & 2 & Rar & 2 \\
\hline Total & 9 & 55 & & 42 & & 209 & & 306 \\
\hline
\end{tabular}

D=dominância: Rar - rara, Eve - eventual, Sub - subdominante, Dom - dominante, Eud - eudominante.

de pesticidas e de roçadas, por ser considerado uma variedade em declínio.

A família de ácaros mais abundante foi Tydeidae, corroborando os resultados de JOHANN et al. (2009) e KLOCK et al. (2011), os quais registraram, respectivamente, 185 e 171 tideídeos. Lorryia sp. foi a única morfoespécie considerada eudominante em todos os vinhedos em Caxias do Sul. A alta abundância dessa morfoespécie em Caxias do Sul pode estar relacionada à sua alimentação diversificada, constituída por fungos, pólen, e por apresentar-se, eventualmente, com hábito fitófago (MORAES \& FLECHTMANN, 2008). Outro fator que deve ser considerado é a alta incidência de doenças fúngicas nos vinhedos de Caxias do Sul, o que poderia fornecer fonte abundante de alimento a essa espécie, em comparação aos vinhedos de Sant'Ana do Livramento. Os tideídeos podem ainda servir como alimento alternativo para os fitoseídeos (STRICKLER et al., 1987, McMURTRY \& CROFT, 1997, FERLA \& MORAES, 1998). Dessa forma, provavelmente, Lorryia sp. teria possibilitado a manutenção dos predadores nos vinhedos de Caxias do Sul, quando suas presas preferenciais apresentavam baixas densidades.

Os ácaros predadores da família Phytoseiidae constituíram o segundo grupo em abundância (110 ind.), porém apresentaram maior riqueza de espécies (sete). KLOCK et al. (2011) registraram Phytoseiidae como a família com maior riqueza de espécies apresentando igualmente sete espécies. Entre os fitoseídeos, em ambas regiões, T. aripo foi o mais abundante, sendo registrado em todas as plantas da vegetação espontânea. Em Sant'Ana do Livramento esteve associado principalmente à variedade Cabernet Sauvignon e em Caxias do Sul à Isabel (Tabelas 1 e 2). KLOCK et al. (2011) registraram um único espécime ocorrendo em Senecio sp. (Asteraceae) em vinhedo da variedade Chardonnay em Bento Gonçalves. Neoseiulus californicus foi registrado unicamente em Sant'Ana do Livramento, associado às duas variedades de Vitis vinifera (L.) De VILLIERS \& PRINGLE (2011) registraram $N$. californicus como uma das três espécies de fitoseídeos mais frequentes e abundantes,ocorrendo em $\boldsymbol{R}$. raphanistrum durante três anos de amostragens em vinhedos na África do Sul. Essa espécie pode alimentarse tanto de artrópodes em geral e ácaros tetraniquídeos, bem como de pólen, ocupando uma situação intermediária entre os Tipos II e III (McMURTRY \& CROFT, 1997). Além disso, pode se estabelecer no agroecossistema antes dos ácaros fitófagos e mantêlos em níveis muito baixos (PRINGLE \& HEUNIS, 2006). Isto pode ser corroborado pelo pequeno número de Brevipalpus phoenicis (Geijskes, 1939) registrado nas plantas da vegetação espontânea (Tabela 2). 
Tabela 2 - Acarofauna presente na vegetação espontânea em vinhedos das cultivares Cabernet Sauvignon (CS) e Merlot (M) em Sant’Ana do Livramento, Rio Grande do Sul, Brasil. ( $\mathrm{n}=3120$ plantas).

\begin{tabular}{|c|c|c|c|c|c|c|}
\hline Família/Vegetação & Família/Ácaro & Espécie/Ácaro & CS & $\mathrm{D}$ & M & $\mathrm{D}$ \\
\hline Asteraceae & Phytoseiidae & & & & & \\
\hline \multirow[t]{11}{*}{ Bidens pilosa } & & Neoseiulus californicus & 9 & Dom & 2 & Sub \\
\hline & & Neoseiulus tunus & - & & 2 & Sub \\
\hline & & Typhlodromalus aripo & 20 & Eud & 1 & Eve \\
\hline & & Typhlodromalus peregrinus & 4 & Sub & 2 & Sub \\
\hline & Tarsonemidae & & & & & \\
\hline & & Steneotarsonemus sp. & 4 & Sub & 7 & Eud \\
\hline & & Tarsonemus sp.1 & 2 & Eve & 7 & Eud \\
\hline & Tenuipalpidae & & & & & \\
\hline & & Brevipalpus phoenicis & 1 & Rar & 2 & Sub \\
\hline & Tydeidae & & & & & \\
\hline & & Lorryia sp. & - & & 1 & Eve \\
\hline brassicaceae & Phytoseiidae & & & & & \\
\hline \multirow[t]{2}{*}{$\begin{array}{l}\text { Raphanus } \\
\text { raphanistrum }\end{array}$} & & N. californicus & 3 & Sub & 2 & Sub \\
\hline & & T. aripo & 1 & Rar & - & \\
\hline Malvaceae & Phytoseiidae & & & & & \\
\hline \multirow[t]{14}{*}{ Sida rhombifolia } & & N. californicus & 7 & Dom & - & \\
\hline & & N. tunus & 1 & Rar & 1 & Eve \\
\hline & & T. aripo & 8 & Dom & 1 & Eve \\
\hline & Tarsonemidae & & & & & \\
\hline & & Fungitarsonemus sp. & - & & 6 & Eud \\
\hline & & Steneotarsonemus sp. & 30 & Eud & 8 & Eud \\
\hline & & Tarsonemus sp.1 & 2 & Eve & 4 & Dom \\
\hline & & Tarsonemus sp.2 & - & & 9 & Eud \\
\hline & Tenuipalpidae & & & & & \\
\hline & & B. phoenicis & 2 & Eve & - & \\
\hline & Tydeidae & & & & & \\
\hline & & Lorryia sp. & 1 & Rar & - & \\
\hline & Winterschmidtiidae & & & & & \\
\hline & & Czenspinskia sp. & 1 & Rar & - & \\
\hline Solanaceae & Phytoseiidae & & & & & \\
\hline \multirow[t]{8}{*}{ Solanum americanum } & & N. californicus & 1 & Rar & - & \\
\hline & & T. aripo & 2 & Eve & - & \\
\hline & Tarsonemidae & & & & & \\
\hline & & Steneotarsonemus sp. & 1 & Rar & - & \\
\hline & Tenuipalpidae & & & & & \\
\hline & & B. phoenicis & 1 & Rar & - & \\
\hline & Winterschmidtiidae & & & & & \\
\hline & & Czenspinskia sp. & 12 & Eud & - & \\
\hline Total & & 11 & 113 & & 55 & \\
\hline
\end{tabular}

D=dominância: Rar - rara, Eve - eventual, Sub - subdominante, Dom - dominante, Eud - eudominante.

Ciência Rural, v.43, n.9, set, 2013. 
Neoseiulus tunus, foi coletada em todas as variedades de videira em Caxias do Sul e em Sant'Ana do Livramento. De acordo com McMURTRY \& CROFT (1997), N. tunus pertence ao grupo III de predadores, os quais podem sobreviver e reproduzir-se sobre as mais diversas fontes de alimento, inclusive pólen, o que deve favorecer a sua ocorrência na vegetação espontânea. A espécie T. peregrinus (Tabela 2) é registrada pela primeira vez no estado do Rio Grande do Sul, tendo sido coletada exclusivamente em $\boldsymbol{B}$. pilosa, possivelmente, devido ao pólen provido pela planta. De acordo com FOULY et al. (1995), essa espécie é generalista e apresenta alta taxa de longevidade e fecundidade quando alimentada com pólen de Quercus virginiana Miller e Typha latifolia L.. Amblyseius herbicolus, apesar de já ter sido registrada em várias plantas nativas e cultivadas no Brasil (MORAES et al., 2004), é relatada pela primeira vez em plantas associadas a Vitis sp. no Brasil, tendo sido registrado exclusivamente em Caxias do Sul, na variedade Isabel (Tabela 1).

Entre os fitófagos, a família mais abundante foi Tarsonemidae (82ind.), sendorepresentadaporquatro morfoespécies (Tabelas 1 e 2). No estudo de KLOCK et al. (2011), essa família também foi a mais representativa entre os ácaros fitófagos. Tarsonemidae apresenta espécies com hábitos alimentares diferenciados, desde aquelas que se alimentam de fungos, algas e plantas superiores até espécies predadoras e parasitas de insetos (MORAES \& FLECHTMANN, 2008). O tarsonemídeo mais abundante foi Steneotarsonemus sp., o qual não foi registrado apenas na variedade Sémillon em Caxias do Sul. A morfoespécie Fungitarsonemus sp. foi registrada exclusivamente na variedade Merlot em Sant’Ana do Livramento. Esse gênero apresenta hábito alimentar pouco conhecido, mas, de acordo com FLECHTMANN \& MORAES (2001), pode servir como fonte de alimento alternativo, favorecendo a presença de inimigos naturais em agroecossistemas. Estes dois tarsonemídeos não foram coletados na vegetação espontânea de vinhedos em Bento Gonçalves, Candiota e Encruzilhada do Sul (KLOCK et al., 2011 e JOHANN et al., 2009). As duas morfoespécies de Tarsonemus sp. ocorreram exclusivamente nos vinhedos de Sant'Ana do Livramento. A segunda família em abundância entre os fitófagos, Tenuipalpidae, foi representada por B. phoenicis, o qual foi considerado subdominante em Caxias do Sul e raro em Sant'Ana do Livramento. Esse resultado corrobora o encontrado por KLOCK et al. (2011), os quais registraram um exemplar deste ácaro em $\boldsymbol{S}$. americanum em vinhedo de Cabernet Sauvignon em Candiota, RS, sendo considerado raro. Brevipalpus phoenicis é considerada de grande importância econômica no Brasil por ser transmissora de vírus em culturas como citros e café (MORAES \& FLECHTMANN, 2008), apesar de não ser referida como causadora de danos econômicos na cultura da videira (FLECHTMANN, 1979).

O gênero Czenspinskia, único representante de Winterschmidtiidae registrado, pode utilizar-se de néctar como fonte de alimento (PEMBERTON, 1993) e esteve associado exclusivamente a vinhedos de Cabernet Sauvignon em Sant'Ana do Livramento, ocorrendo em Solanum americanum (Mill.), a qual é considerada uma planta néctar-polinífera, muito visitada por abelhas (JUNIOR et al., 2007).

O maior número de ácaros predadores em relação aos ácaros fitófagos presentes na vegetação espontânea de vinhedos em Caxias do Sul e Sant'Ana do Livramento corrobora os resultados de JOHANN et al. (2009) e KLOCK et al. (2011), em vinhedos no RS. Esses dois estudos têm associado a alta abundância de predadores, em especial de fitoseídeos, à presença de espécies de ácaros fitófagos como Calepitrimerus vitis (Nalepa, 1905), em vinhedos sem a aplicação de pesticidas. Entretanto, no presente estudo, não foi registrada alta abundância de espécies fitófagas, podendo-se atribuir a menor abundância destas à aplicação de acaricidas. O controle químico de ácaros nos vinhedos estudados em Caxias do Sul e Sant'Ana do Livramento é realizado preventivamente, sem monitoramento prévio das populações, e sem o conhecimento do nível de dano para qualquer espécie.

Relação entre acarofauna, plantas da vegetação espontânea e variedades de videira em Sant'Ana do Livramento

Dentre as quatro espécies da vegetação espontânea coletadas nos vinhedos de Sant'Ana do Livramento, Sida rhombifolia apresentou maior abundância de indivíduos (81), com diferença significativa na abundância de ácaros entre as espécies vegetais $(\mathrm{F}=3,408 ; p=0,03)$ e a maior riqueza de espécies (Tabela 2). Isso se deve, possivelmente devido à complexidade estrutural e densidade foliar apresentada pela planta, oferecendo microclima e abrigo adequados à acarofauna. Um fator que pode ter influenciado na maior abundância e riqueza de espécies de ácaros é a maior ocorrência de $\boldsymbol{S}$. rhombifolia durante as ocasiões amostrais. As práticas de manejo empregadas nos vinhedos, como roçadas e aplicações de herbicidas fizeram com que as abundância das espécies vegetais variassem ao longo das ocasiões de amostragem. A espécie vegetal que apresentou o menor número de indivíduos (seis) e riqueza de espécies de ácaros associados (dois) foi $\boldsymbol{R}$. raphanistrum, resultado 


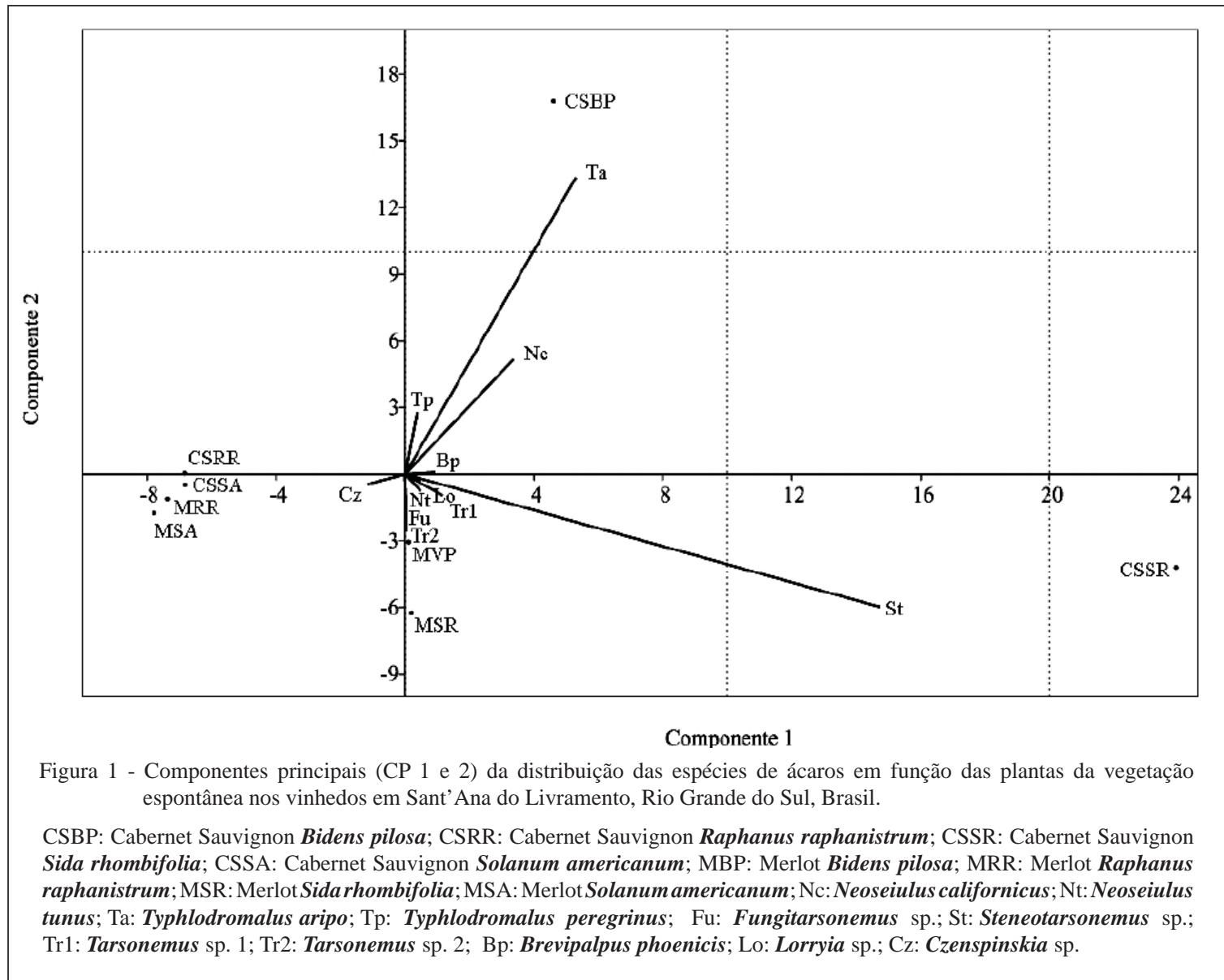

que corrobora o estudo de SANTOS (2011) o qual identificou um total de 32 espécies de ácaros, sendo apenas quatro espécies registradas em $\boldsymbol{R}$. raphanistrum, em um levantamento de acarofauna em 167 espécies de plantas espontâneas de vinhedos em Setúbal, Portugal.

Através da análise de Componentes Principais (Figura 1), podemos visualizar que o conjunto de vinhedos de ambas as variedades que incluem as plantas $\boldsymbol{R}$. raphanistrum e $\boldsymbol{S}$. americanum encontramse agrupados, provavelmente, em razão da baixa riqueza e abundância de espécies de ácaros nesses locais (Tabela 2). O fitoseídeo T. aripo está associado à presença de B. pilosa em ambos os vinhedos, com maior incidência em Cabernet Sauvignon. Por sua vez, a morfoespécie Steneotarsonemus sp. parece estar relacionada à $\boldsymbol{S}$. rhombifolia, sendo registrada nos vinhedos de ambas variedades.

\section{CONCLUSÃO}

Nas plantas da vegetação espontânea associadas aos vinhedos em Caxias do Sul e Sant’Ana do Livramento, está presente um total de
14 espécies de ácaros, sendo sete delas predadoras. O maior número de ácaros ocorre em Caxias do Sul, entretanto, a riqueza de espécies é maior em Sant'Ana do Livramento. Sida rhombifolia L. é a espécie da vegetação espontânea que reúne o maior número de ácaros. A acarofauna presente nos nove vinhedos amostrados caracteriza-se pela predominância de Lorryia sp., seguida por Typhlodromalus aripo e Steneotarsonemus sp.. Registra-se Amblyseius herbicolus pela primeira vez em plantas associadas à Vitis sp. no Brasil e Typhlodromalus peregrinus tem seu primeiro registro para o estado do Rio Grande do Sul.

\section{AGRADECIMENTOS}

Os autores agradecem à Dra. Ilsi Boldrini (UFRGS), pela identificação das espécies botânicas; ao Dr. Andre Luis Matioli e ao Dr. Jefferson Mineiro (Instituto Biológico de São Paulo), ao Dr. Noeli Juarez Ferla (UNIVATES), à Mestre Liana Johann (PUCRS, UNIVATES) e a Matheus dos Santos Rocha (UNIVATES), pelas identificações; à Eng. ${ }^{a}$ Agr. ${ }^{a}$ Nicole de Carvalho Barros, aos graduandos de Agronomia da UFRGS, Manuela Sulzbach, Pedro Augusto Veit e Leonardo Sobrosa Rosito e à Biól. ${ }^{a}$ Maria Rita Poeta, pelo apoio durante as amostragens e processamento do

Ciência Rural, v.43, n.9, set, 2013. 
material; ao Conselho Nacional de Desenvolvimento Científico e Tecnológico (CNPq) (Processo 131157/2009-4) e aos revisores anônimos, pelas sugestões e comentários ao manuscrito.

\section{REFERÊNCIAS}

ALTIERI, M.A. et al. Manipulating vineyard biodiversity for improved insect pest management: case studies from northern California. International Journal of Biodiversity Science and Management, v. 1, p. 1-13, 2005. Disponível em: <http:// agroeco.org/wp-content/uploads/2010/09/Int.J.Biodiv-Mgmt. pdf $>$. Acesso em: 15 set. 2012

AYRES et al. Bioestat 4.0: Aplicações estatísticas nas áreas das ciências biológicas e médicas. Belém: Sociedade Civil Mamirauá, 2005. 334p.

BABAR, Z. et al. Does agroforestry affect phytoseiid mite communities in vineyards in the south of France? In: SABELIS, M.W.; BRUIN, J. Trends in Acarology: Proceedings of the $12^{\text {th }}$ International Congress. Dordrecht: Springer Science+Business Media B.V., 2010. p. 409 - 412. Disponível em: <http://link. springer.com/book/10.1007/978-90-481-9837-5/page/1/\#page-1> Acesso em: 30 ago. 2012. doi: 10.1007/978-90-481-9837-5_66.

CHANT, D.A.; McMURTRY, J.A. Illustred keys and diagnose for the genera and subgenera of the Phytoseiidae of the world. Michigan: Indira Publishing House, 2007. 220p.

De VILLIERS, M., PRINGLE, K.L. The presence of Tetranychus urticae (Acari: Tetranychidae) and its predators on plants in the ground cover in commercially treated vineyards. Experimental and Applied Acarology, n. 53, p. 121-137, 2011. Disponível em: $<$ http://link.springer.com/article/101007\%2Fs10493-010-93917?LI=true\#page-1>. Acesso em: 15 fev. 2013. Dói: 10.1007/ s10493-010-9391-7.

DUSO, C. et al. Biological control of mites in European vineyards and the impact of natural vegetation. In: SABELIS, M.W.; BRUIN, $\mathrm{J}$. Trends in Acarology: Proceedings of the $12^{\text {th }}$ International Congress. Dordrecht: Springer Science+Business Media B.V., 2010.p. 399 - 407. Disponível em :<http://link.springer.com/ book/10.1007/978-90-481-9837-5/page/1/\#page-1>.Acesso em: 30 ago. 2012. doi: 10.1007/978-90-481-9837-5-65.

FERLA, N.J.; BOTTON, M. Ocorrência do ácaro vermelho europeu Panonychus ulmi (Koch) (Tetranychidae) associado à cultura da videira no Rio Grande do Sul, Brasil. Ciência Rural, v. 38, n. 6, p. 1758 - 1761, 2008. Disponível em: <http://www.scielo. br/pdf/cr/v38n6/a42v38n6.pdf>. Acesso em: 18 set. 2012.

FERLA, N.J.; MORAES, G.J. Ácaros predadores em pomares de maçã no Rio Grande do Sul. Anais da Sociedade Entomológica do Brasil, v. 27, n. 4, p. 649 - 654, 1998. Disponível em: <http:// www.scielo.br/pdf/rbzool/v19n4/v19n4a06.pdf >. Acesso em: 19 set. 2012.

FERLA, N.J.; MORAES, G.J. Ácaros predadores (Acari) em plantas nativas e cultivadas do Estado do Rio Grande do Sul, Brasil. Revista Brasileira de Zoologia, v. 19, n. 4, p. 1011-1031. 2002. Disponível em: <http://www.scielo.br/pdf/aseb/v27n4/v27n4a19. pdf>. Acesso em: 19 set. 2012.

FLECHTMANN, C.H.W. Ácaros de importância agrícola. São Paulo: Nobel, 1979. 189p.
FLECHTMANN, C.H.W.; MORAES, G.J. Biodiversidade de ácaros no estado de São Paulo. In: BRANDÃO, R.F.; CANCELLO, E.M. Biodiversidade do estado de São Paulo, Brasil: Síntese do conhecimento ao final do século XX: invertebrados terrestres. São Paulo: FAPESP, 2001. p. 58 - 63. Disponível em: <http://www. biota.org.br/pdf/v5cap06.pdf > . Acesso em: 21 set. 2012.

FOULY, A.H. et al. Effects of diet on the biology and life tables of Typhlodromalus peregrinus (Acari: Phytoseiidae). Environmental Entomology, v. 24 , n. 4, p. 870-874, 1995. Disponível em: <http://esa.publisher.ingentaconnect.com/content esa/event/1995/00000024/00000004art00012>. Acesso em: 31 out. 2012.

FRIEBE, B. Zur biologie eines buchenwaldbodens: 3. Die kaferfauna. Carolinea, Karlshue, v. 41, n. 1, p. 45-80, 1983.

HAMMER, O.; HARPER, D. A. T.; RYAN, P. D. Past: paleontological statistics: version 1.37. Disponível em: <http:// folk.uio.no/ohammer/past>. Acesso em: 23 jan. 2013.

JEPPSON, L.R. et al. Mites injurious to economic plants. Berkeley: University of California, 1975. 614p.

JOHANN, L. et al. Acarofauna (Acari) associada à videira (Vitis vinifera L.) no Estado do Rio Grande do Sul. Biociências, v.17, n.1, p. 1-19, 2009. Disponível em: <http://revistaseletronicas. pucrs.br/ojs/index.php/fabio/article/viewFile/4227/4756>. Acesso em: 21 set. 2012

JUNIOR, H.J.B. et al. Plantas visitadas por Apoidea (Hymenoptera) na região de Cacaria, município de Piraí, RJ. Revista Brasileira de Biociências, v.5, supl. 2, p. 1110-1112, 2007. Disponível em: <http://www.ufrrj.br/abelhanatureza/paginas/monografias_ dissertações?monografia_mata_atlantica.pd>. Acesso em: 1 nov. 2012

KLOCK, C.L. et al. Mitefauna (Arachnida: Acari) associated grapevine, Vitis vinifera L. (Vitaceae), in the municipalities of Bento Gonçalves and Candiota, Rio Grande do Sul, Brazil. Check List, v. 7, I. 4, p. 522 - 536, 2011. Disponível em: <http://www. checklist.org.br/getpdf?SL007-11>. Acesso em: 21 set. 2012.

KÖPPEN, W. Climatologia. Cidade do México: Fondo de Cultura, 1948. p. $152-192$

KRANTZ, G.W.; WALTER, D.E. A Manual of Acarology. Lubbock: Texas Tech University Press, 2009. 807p.

McMURTRY, J.A.; CROFT, B.A. Life-styles oh phytoseiidae mites and their roles in biological control. Annual Review of Entomology, v. 42, p. 291-321. 1997. Disponível em:<http://www. annualreviews.org> . Acesso em: 13 mai. 2011.

MONTEIRO, L.B. et al. Efeito do manejo de plantas daninhas sobre Neoseiulus californicus (Acari: Phytoseiidae) em pomar de macieira. Revista Brasileira de Fruticultura, v. 24, n. 3, p. 680 - 682, 2002. Disponível em: <http://www.scielo.br/pdf/rbf/ v24n3/15111.pdf>. Acesso em: 29 agost. 2012.

MORAES, G.J.; FLECHTMANN, C.H.W. Manual de Acarologia Acarologia básica e ácaros de plantas cultivadas no Brasil. Ribeirão Preto: Holos Editora, 2008.288p.

MORAES, G.J. et al. A revised catalog of the mite family Phytoseiidae. Zootaxa, n. 434, p. 1- 494, 2004. 
SANTOS, P.T.M. Acarofauna da vinha e infestantes em zonas edafoclimáticas diferentes na região de Setúbal. 2011. 111 f. Dissertação (Mestrado em Engenharia Agronomica) - Instituto Superior de Agronomia, Universidade Técnica de Lisboa. Disponível em:<http://hdl.handle.net/10400.5/4173>. Acesso em: 15 fev. 2013

SIQUEIRA, P.R.E. et al. Flutuação populacional do ácaro-daferrugem-da-videira em vinhedo comercial em Candiota, RS, com diferentes métodos de amostragem. Ciência Rural, v.31, n.9, p.1489-1495, 2011. Disponível em: <http://www.scielo.br/pdf/cr/ v41n9/a10811cr4002.pdf>. Acesso em: 21 set. 2012.
STRICKLER, K.N. et a. Mite (Acari) species composition in Michigan apple orchards. Environmental Entomology, v. 16, n. 1,p. 30-36, 1987. Disponível em: < http://esa.publisher.ingentaconnect. com/content/esa/envent/1987/00000016/00000001/art00005>. Acesso em: 21 set. 2012.

TIXIER, M.-S. et al. Colonization of Languedoc vineyards by phytoseiid mites (Acari:Phytoseiidae): influence of wind and crop environment. Experimental and Applied Acarology, v. 22, n. 9, p. 523 -542, 1998. Disponível em: < http://www.springerlink. com/content/q14134888jj8w585/>. Acesso em: 21 set. 2012. doi: 10.1023/A:1006085723427. 